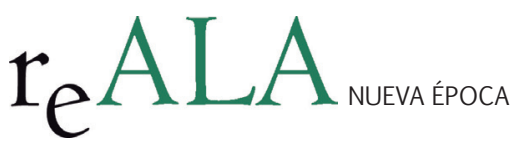

REALA, n 3, enero-junio 2015 ISSN: 1989-8975

DOI: http://dx.doi.org/10.24965/reala.voi3.10250

\title{
La gestión del ciclo integral del agua: los costes, su recuperación y las buenas prácticas. El caso de Gipuzkoa'
}

\author{
Ander Ibarloza Arrizabalaga \\ Profesor Ayudante de Facultad de Ciencias Económicas y Empresariales, Universidad del País Vasco/Euskal Herriko Unibertsitatea (UPVIEHU) \\ ander.ibarloza@ehu.es \\ Eduardo Malles Fernández \\ Profesor Doctor Titular de Escuela Universitaria de Estudios Empresariales, Universidad del País Vasco/Euskal Herriko Unibertsitatea (UPVIEHU) \\ eduardo.malles@ehu.es \\ Itziar Azkue Irigoien \\ Profesora Doctora Titular de Escuela Universitaria de Estudios Empresariales, Universidad del País Vasco/Euskal Herriko Unibertsitatea (UPVIEHU)
} itziar.azkue@ehu.es

\section{Resumen}

Nuestro estudio tiene como objetivo analizar el ciclo integral del agua como servicio básico en las administraciones locales, haciendo especial hincapié en los sistemas de costes de su prestación, así como su repercusión vía tasas al consumidor final y la influencia que tienen las buenas prácticas en la gestión del servicio.

Para alcanzar ese objetivo se analizará su funcionamiento en el Territorio Histórico de Gipuzkoa estudiando el entorno en el que se presta ese servicio tanto desde el ámbito normativo como en el análisis de las distintas etapas del ciclo integral, diferenciando el abastecimiento y el saneamiento del agua y la organización administrativa que se ha creado para poder proporcionar ese servicio.

Palabras clave

Directiva Marco del Agua; Servicio de Aguas; Costes y Tasas; Administración Local; Buenas Prácticas.

\section{The management of the water provision: costings, recovery and good practices. The case of Gipuzkoa}

Abstract

The current study aims to analyze the water provision as a basic service in local government service, with particular emphasis on costing systems. The financial implications for the end-users to recover costs through the taxation system and the influence of good practice in service management are contrasted.

Firstly, in order to achieve this objective, we analyze the organization of water provision in Gipuzkoa, focusing on the legal and technical environments in which the service is provided. Subsequently, we analyzed different stages of the complete cycle (water supply and water sanitation) and each water provider organization.

Keywords

Water Framework Directive; Water Service; Costing and Taxes; Local Administration; Good Practices

1 Este articulo ha sido realizado en el marco del Proyecto de Investigación NUPV 13/09 “Diseño de un sistema de costes en los servicios públicos municipales de Gipuzkoa y su repercusión en el establecimiento de tasas y precios públicos" de la UPV/EHU y el Convenio de colaboración para el "Diseño de un sistema de costes de los Servicios Públicos Municipales de Gipuzkoa" entre la UPV/EHU y la Diputación Foral de Gipuzkoa (BOG, n 130, 09-07-2013). 


\section{INTRODUCCIÓN}

Las entidades locales prestan a sus habitantes determinados servicios que se pueden catalogar como básicos, fundamentales o de interés general, y que persiguen satisfacer unas condiciones de bienestar social mínimos. El abastecimiento de agua potable y el saneamiento de aguas residuales son dos de los servicios esenciales que presta un municipio a su ciudadanía, y son, al mismo tiempo, unos servicios particulares por la forma en la que se ofrecen así como por el análisis de costes que acarrean las distintas actividades y su recuperación económica y regeneración posteriores.

Para poder llevar a cabo la prestación de estos servicios, las entidades locales disponen de unos recursos y una financiación limitados que provienen de la actividad económica y de sus agentes, como son las empresas y la ciudadanía, mediante las diferentes vías tributarias, además de las transferencias supramunicipales y las subvenciones. La actual crisis, el descenso de la actividad económica y la consiguiente reducción de recursos económicos y financieros en las entidades locales repercuten en que los servicios se tengan que ajustar aún más. Resulta necesario gestionar eficientemente estos servicios para intentar mantener en el tiempo, con criterios de sostenibilidad, los aspectos cualitativos y cuantitativos vinculados a los mismos.

El objetivo de este trabajo es el de mostrar el marco en que se desenvuelve este servicio público esencial en la vida de las personas, incidiendo en el cálculo de los costes y su repercusión, vía tasas, a las personas usuarias de ese servicio. Para ello se acometerá la tarea de revisar el carácter público del servicio y la normativa estatal y europea sobre la materia, y en especial aquella relativa a los costes y su influencia en la fijación de las tarifas. Se incidirá en la Directiva 2000/60/CE², conocida como Directiva Marco del Agua (en adelante, DMA) como instrumento normativo que pretende dirigir a las entidades prestadoras del servicio hacia las buenas prácticas y la transparencia en su gestión. Asimismo se especificarán las fases que conlleva el ciclo integral del agua, diferenciando tanto las actividades en Alta como en Baja, distinguiendo a su vez el abastecimiento y saneamiento de las aguas así como los distintos modelos organizativos que surgen para prestar y gestionar mejor ese servicio, subrayando algunas de las buenas prácticas llevadas a cabo en el ámbito territorial al que dirigimos nuestro estudio, el Territorio Histórico de Gipuzkoa. Para finalizar, se abordará el análisis del cálculo de los costes de este servicio, observando los aspectos que se tienen en consideración al definirlos, la metodología y la forma de fijar las tasas a la ciudadanía usuaria y la relación existente entre los costes y la recuperación de los mismos a través de las tasas establecidas.

\section{EL AGUA COMO SERVICIO PÚBLICO OBLIGATORIO}

La normativa estatal determina que el correcto cumplimiento de las competencias municipales requiere que los municipios presten los servicios considerados obligatorios o mínimos. Así, la Ley reguladora de las Bases del Régimen Local'3 (en adelante, LRBRL), modificada por la Ley de racionalización y sostenibilidad de la Administración Local $^{4}$, en su artículo 26, cataloga entre este tipo de servicios los siguientes ${ }^{5}$ : alumbrado público, cementerio, recogida de residuos, limpieza viaria, abastecimiento domiciliario de agua potable, alcantarillado, acceso a los núcleos de población y pavimentación de las vías públicas.

En nuestro caso, la captación, abastecimiento, recogida y saneamiento de las aguas, es decir, el ciclo integral del agua, es un servicio esencial para la ciudadanía y de gran relevancia económica para la administración pública, lo que justifica el interés en la realización de un estudio como el que se presenta.

Es importante diferenciar este ciclo integral del ciclo natural del agua y mantener una visión global, de modo que sea posible valorar el impacto de las acciones del ser humano en dicho ciclo natural y el daño irreversible en los ecosistemas y el medioambiente. Estas acciones no provienen únicamente del abastecimiento de agua potable o del ciclo integral del agua, sino que el desarrollo de algunas actividades territoriales también inciden sobre ello: actividades, entre otras, del sector agropecuario (deforestación, técnicas de riego, pesticidas, excedentes de purines...), del sector industrial (polígonos industriales, carreteras, desechos...), del sector comercial (grandes superficies comerciales, aparcamientos...), y del sector turístico (complejos residenciales, puertos, bahías,

2 Directiva 2000/60/CE del Parlamento Europeo y del Consejo de 23 de octubre de 2000 por la que se establece un marco comunitario de actuación en el ámbito de la política de aguas, (Diario Oficial de las Comunidades Europeas 22/12/2000).

3 Ley 7/1985, de 2 de abril, reguladora de las Bases del Régimen Local, (BOE núm. 80 de 03 de Abril de 1985).

4 Ley $27 / 2013$, de 27 de diciembre, de racionalización y sostenibilidad de la Administración Local, (BOE núm. 312 de 30 de Diciembre de 2013).

5 Además de éstos hay otros servicios que las entidades locales deben prestar en municipios con población superior a 5.000, 20.000 y 50.000 habitantes. 
canalización de los ríos... ), sin dejar a un lado, los avances de áreas urbanas invadiendo las riberas ${ }^{6}$. Es evidente que el debate sobre el ciclo natural del agua podría abarcar un campo mucho más amplio en relación con el desarrollo de las actividades territoriales mencionadas, pero no será objeto de estudio en este trabajo por su amplitud.

Efectivamente tenemos la capacidad tecnológica y técnica de ejecución de todas estas actividades y la política de las últimas décadas ha sido la de construir en vez de la de promover una correcta gestión. Tal y como ha sucedido con otros recursos medioambientales, el desarrollo de la población humana no ha ido acorde a la capacidad natural de servicio y regeneración de este recurso limitado y, en consecuencia, se comenzó a domesticar, dominar o contener el abastecimiento del agua. Para ello se construyeron presas, encauzamientos, canalizaciones, embalses artificiales, estaciones de tratamiento de agua potable (ETAP), y se procedió a los trasvases y a la desalación del agua del mar, con el objetivo de maximizar la producción y garantizar el abastecimiento, a costa de sustraer al medioambiente de este recurso tan valioso, necesario y básico para su supervivencia, reduciendo drásticamente los caudales e incluso eliminando los cauces de los ríos.

Toda esta demanda hídrica evidenció, en un periodo de tiempo relativamente corto, la contaminación de este recurso en los ríos y la muerte biológica que se producía en su transcurso, arrebatando al medioambiente su capacidad de regeneración. El brutal deterioro medioambiental concienció a la sociedad de la necesidad de asumir el coste del saneamiento de las aguas utilizadas y contaminadas, planteándose para ello la construcción de canales de recogida de aguas residuales y de Estaciones de Depuración de Aguas Residuales (EDAR) en el Plan Hidrológico Nacional del 2000 (PHN 2000). Aún así, hay quien cataloga este plan como una mera propuesta de realización de obras hidráulicas, basadas en una memoria económica, que infravaloraba los costes de las obras, y una memoria ambiental, que rehúye la realización de los daños ambientales ocasionados.

La política desarrollada en materia hidrológica en España derivó en que se convirtiera en el país con más presas por kilómetro cuadrado y habitante ${ }^{8}$. Así la capacidad de los embalses se vio duplicada pero también se incrementaron, incluso en mayor medida, las demandas de agua. Para atender este aumento de demanda se realizan, además, trasvases entre cuencas o desalinizaciones de agua y, por supuesto, todo esto tiene un coste económico y medioambiental. Es necesario, por lo tanto, analizar estas demandas y analizar si se hace un uso sostenible de este recurso limitado. Y, por consiguiente, las instituciones públicas también deberán plantearse la cuestión de determinar el capital que será necesario destinar a la construcción, mantenimiento o reposición de estas infraestructuras 9 . Y es que el agua potable es uno de los ejemplos recurrentes de recurso de primera necesidad cuyo valor no se aprecia hasta que no se tiene, debido en gran medida a que la demanda actual está cubierta y tiene una tarifa contenida.

Este servicio, en España, está sujeto a unas tasas que no deben superar el coste del servicio prestado para poder asegurar, de esta manera, el derecho de la ciudadanía a su acceso, entre otras razones, por ser una prestación de servicio ofrecida en régimen de monopolio ${ }^{10}$ y ser esencial para la vida de las personas. Según la normativa aplicable a la materia,

“el importe de las tasas por la prestación de un servicio o por la realización de una actividad no podrá exceder, en su conjunto, del coste real o previsible del servicio o actividad de que se trate" y "se tomarán en consideración los costes directos e indirectos, inclusive los de carácter financiero, amortización del inmovilizado y, en su caso, los necesarios para garantizar el mantenimiento y un desarrollo razonable del servicio"11

El panorama descrito no es exclusivo del Estado español y muestra evidencias en todos los países del entorno. Así, la Unión Europea en un intento de regular el buen uso del agua, ha desarrollado una conceptualización del mismo en la DMA, al señalar que "el agua no es un bien comercial como los demás, sino un patrimonio que hay que proteger, defender y tratar como tal". Esta y otras directivas han sido objeto de incumplimiento por parte de los Estados miembros de la Unión Europea y, por ende, la cuestión se ha judicializado. En este sentido, y en lo que a España se refiere, sirvan como ejemplo las condenas recibidas por parte del Tribunal de Justicia de la UE en

MORAL (2009) y JUÁREZ (2008).

NAREDO (2006).

ARROJO (2004).

DOMÈNECH (2011).

ORDÓÑEZ y BRU (2003).

Art. 19.2 y 3 de la Ley 8/1989, de 13 de abril, de Tasas y Precios Públicos, (BOE de 15 de Abril de 1989). 
2011'2 , señalando que España era culpable del incumplimiento de la Directiva 91/271/CEE del Consejo, de 21 de mayo de 1991, relativa al tratamiento de las aguas residuales urbanas, y en $2012^{13}$ por no haber iniciado en el año 2008 el procedimiento de información y consulta públicas, ni adoptado (en el 2009) ni presentado (en el 2010) los planes hidrológicos de cuenca a los que obligaba la DMA.

\section{LA DIRECTIVA MARCO DEL AGUA, LA RECUPERACIÓN DE COSTES Y LAS BUENAS PRÁCTICAS}

La DMA es el instrumento normativo procedente de la UE que fija los aspectos básicos y objetivos a conseguir por todos los Estados miembros. En este sentido, cada Estado miembro transpone la Directiva a su ordenamiento interno y en el caso del Estado español esta transposición se efectuó a través de la Ley 62/2003, de 30 de diciembre, de medidas fiscales, administrativas y de orden social que incorpora, a través de su artículo 129, la modificación del Texto Refundido de la Ley de Aguas ${ }^{14}$ que introducía la DMA al Derecho interno español. Posteriormente, en desarrollo de la anterior normativa estatal, se ha aprobado el Reglamento de Planificación Hidrológica ${ }^{15}$. En cuanto al marco normativo de la Comunidad Autónoma del País Vasco (en adelante, CAPV), normativa que será de aplicación al Territorio Histórico de Gipuzkoa, debe mencionarse la Ley 1/2006 de Aguas ${ }^{16}$.

La DMA fija, en su artículo 9, que los Estados miembros de la UE deben tener en cuenta dos principios básicos:

1.- El principio de la recuperación de los costes de los servicios relacionados con el agua, considerando además de los costes directos e indirectos imputables, los costes medioambientales y los relativos a los recursos naturales, los ingresos por los servicios del agua, el nivel actual de recuperación de costes y las inversiones previstas.

2.- El principio de que quien contamina paga.

Con estos principios se persigue vincular el coste al precio, sensibilizar y concienciar a la ciudadanía de un uso eficiente y sostenible de los recursos hídricos, ya que la política de precios es una de las estrategias más eficaces de valoración e influyentes en el consumo. El abastecimiento de agua potable sigue siendo cuasi-gratuito en algunos municipios $^{17}$, aunque cada vez quedan menos, y han sido numerosos los estudios que afirman que los servicios cuasi-gratuitos o subvencionados total o parcialmente, si no van acompañados de otras medidas de sensibilización y concienciación, son peor valorados y consecuentemente malgastados ${ }^{18}$.

A lo anterior hay que sumar otro elemento que es la llave de la conducta futura o de la ejecución de la acción: la confianza' ${ }^{19}$. Es necesario atajar en la medida de lo posible dos hechos que repercuten en ella, aunque se trate de un tema tangencial con respecto al servicio de aguas. Nos referimos al tema de los altos índices registrados en la percepción de la corrupción ${ }^{20}$ y a las campañas de descredito suscitadas en el transcurso de esta crisis en relación con la eficiencia de los recursos de las entidades públicas. Para ello, al margen de otras medidas, será necesario incidir en tres aspectos básicos como son la participación ciudadana, la transparencia y las nuevas formas de rendición de cuentas ${ }^{21}$. Los tres, se citan en leyes directamente relacionadas con la prestación de los distintos servicios públicos locales; así, la LRBRL en su artículo 1 establece que,

“Los Municipios son entidades básicas de la organización territorial del Estado y cauces inmediatos de participación ciudadana en los asuntos públicos, que institucionalizan y gestionan con autonomía los intereses propios de las correspondientes colectividades."

Por su parte la Ley 18/2001 General de Estabilidad Presupuestaria22, en su artículo 2, se refiere a cuatro principios rectores: estabilidad presupuestaria (artículo 3), plurianualidad (artículo 4), transparencia (artículo 5) y eficiencia en la asignación y utilización de recursos públicos (artículo 6). Asimismo, con la largamente esperada Ley 19/2013

\footnotetext{
12 STJUE, de 14 de abril de 2011, Asunto C-343/10, Comisión Europea contra España.

13 STJUE, de 4 de octubre de 2012, Asunto C-403/11, Comisión Europea contra España.

14 Real Decreto Legislativo 1/2001, de 20 de julio, por el que se aprueba el texto refundido de la Ley de Aguas, (BOE núm. 176 de 24 de

15 Real Decreto 907/2007, de 6 de julio, por el que se aprueba el Reglamento de la Planificación Hidrológica, (BOE núm. 162 de 07 de

16 Ley $1 / 2006$, de 23 de junio, de Aguas, (BOPV núm. 137 de 19 de Julio de 2006).

17 MUÑIZ Y GARCÍA (2004).

18 DEL VILLAR (2010).

19 WELCH ET AL. (2005).

20 Primer Informe sobre la lucha contra la corrupción en la UE publicado por la Comisión Europea: Más del 75\% de los ciudadanos europeos, y un 95\% de los españoles, coinciden en que la corrupción está muy extendida en su país de origen. COMISIÓN EUROPEA (2009).

21 KIM ET AL. (2005).

22 Ley 18/2001, de 12 de diciembre, General de Estabilidad Presupuestaria, (BOE núm. 298 de 13 de Diciembre de 2001).
} Julio de 2001). Julio de 2007). 
de transparencia ${ }^{23}$ el Estado español se suma al amplio grupo de Estados miembros de la Unión Europea con una normativa específica y con un instrumento esencial de profundización y regeneración democrática. Ahora bien, no se ajusta a los estándares internacionales en la materia, pues no reconoce el derecho de acceso a la información como parte integrante de la libertad de información y, por ende, como derecho fundamental ${ }^{24}$.

De todo ello podemos extraer que una adecuada prestación del servicio del agua conllevaría el respeto de los aspectos que se señalan a continuación:

- Transparencia y rendición de cuentas: será necesaria una actitud proactiva para la mejora en la eficiencia en el uso del agua por parte de la ciudadanía consumidora, siendo una de las claves la confianza de los usuarios de este servicio. Que la información con respecto al servicio esté ordenada, sea relevante ${ }^{25}$ y esté disponible ${ }^{26}$ es, junto a una correcta gestión, planificación, ejecución y control ${ }^{27}$, uno de los principales factores que influyen en la confianza del usuario y en su actitud proactiva.

- Concienciación: los informes de sostenibilidad de las entidades públicas, aunque todavía no son muy frecuentes ${ }^{28}$, parecen estar relacionados con las actividades estratégicas reales en términos de sostenibilidad y orientados a ampliar la conciencia social sobre los problemas esenciales de sostenibilidad del ciclo integral del agua, sin tratar de tergiversar la realidad ${ }^{29}$. Sin embargo, tal y como sugiere la doctrina académica, en las empresas privadas con fines de lucro, el medioambiente está subordinado al éxito financiero ${ }^{30}$ y los informes de sostenibilidad no abordan las cuestiones básicas de sostenibilidad ${ }^{31,32}$. Además se deben fomentar actividades de formación dirigidas a un consumo cada vez más responsable y adoptar otra serie de medidas que repercutan en la acción. A modo de muestra, la provincia de Málaga fue pionera a finales de 2013 en aplicar un sistema de tramos por habitante que se rige por habitante empadronado en la vivienda. Aunque a priori, todas las partes coinciden en que la asignación de metros cúbicos por habitante empadronado es más justo y puede concienciar sobre el consumo de cada persona y fomentar un uso más responsable, las discrepancias fundamentales estallaron por las cantidades fijadas en los distintos tramos y tarifas.

- Participación ciudadana: siendo como es una cuestión fundamental recogida en la DMA y en la LRBRL, se encuentra estrechamente relacionada con el déficit democrático, con la puesta en valor de aquellos aspectos individuales que influyen en la conciencia social ${ }^{33}$ y con los procesos de adopción de decisiones como el mencionado anteriormente. Aún queda mucho recorrido en la concienciación sobre la importancia del agua como recurso vital y como bien social y medioambiental. Los costes medioambientales o los costes de los recursos naturales, mencionados en la DMA, necesitan de un consenso democrático para ser explicitados y poder así sensibilizar y concienciar de algún modo a la ciudadanía.

- Control: se trata de un instrumento necesario para conocer el desarrollo de los objetivos planteados a consecuencia de las acciones emprendidas y medidas adoptadas. Precisamente, la ausencia de análisis de los resultados obtenidos es una de las carencias más notorias y criticadas en la gestión pública. Aunque la revelación de los indicadores financieros y presupuestarios es relativamente alta, la divulgación de indicadores relativos a la gestión de los servicios públicos locales sigue siendo muy escasa34.

Más allá del establecimiento de principios y pautas para la correcta prestación del servicio del agua, la DMA se refiere de modo explícito al estudio de la recuperación de costes de los servicios de agua. La apuesta de la DMA por las políticas tarifarias tendentes a garantizar la recuperación de costes de los servicios de aguas es clara, pero debido a lo complejo que podría resultar también deja ciertos márgenes para posibles interpretaciones ${ }^{35}$. Por un

23 Ley 19/2013, de 9 de diciembre, de transparencia, acceso a la información pública y buen gobierno, (BOE núm. 295, de 10 de Diciembre de 2013).

24 GUICHOT (2014).

5 PINA ET AL. (2009).

CHAN (2003).

LEE (2008).

MUSSARI Y MONFARDINI (2010).

LARRINAGA Y PÉREZ (2008).

BebBington et Al. (2008).

BALL Y GRUBNIC (2007).

GRAY (2006).

KLUVERS (2010).

ROYO ET AL. (2012)

MORAL Y ARROJO (2003). 
lado están las concreciones de cada uno de los costes y por el otro, el nivel de recuperación de estos costes que evidentemente están directamente relacionados con el establecimiento de las tarifas.

En este sentido y partiendo de que según la Organización Mundial de la Salud (OMS) se requieren entre 50 y 100 litros de agua en unas condiciones mínimas de uso por persona al día para cubrir la mayoría de las necesidades básicas, hay que añadir la repercusión de este servicio en el bienestar social, ya que la higiene tiene una relación directa con la reducción de enfermedades ${ }^{36}$. Es por ello que se debe garantizar este servicio a todos los sectores de la población pero sin perjuicio de poder restablecer este servicio al conjunto general ${ }^{37}$. Es habitual en estos casos disponer de distintas tarifas en función de los consumos y según se vayan incrementando éstos, utilizarlos como sensibilizadores, recuperadores de coste o como penalizadores por despilfarro, uso abusivo o malas prácticas. Ahora bien, el establecimiento de unas tarifas excesivamente laxas supone que las entidades locales deberán recurrir a otros recursos para hacer frente a estos costes y reducirán, de este modo, su capacidad para prestar otros servicios.

La Ley de Tasas y Precios Públicos en su artículo 6 conceptualiza las "tasas" como aquellos tributos cuyo hecho imponible consiste en la utilización privativa o el aprovechamiento especial del dominio público cuando los servicios no se presten o realicen por el sector privado, y en el artículo 19 añade que el importe de las tasas por la prestación de un servicio no podrá exceder, en su conjunto, del coste real o previsible del servicio.

A todo ello hay que añadir que los políticos conocedores del, no siempre acertado, punto de vista de que los incrementos de las tasas suscitan el castigo en votos, suelen ser muy reacios a las subidas de estas tasas. Como consecuencia nos enfrentamos a unas tasas que no han sido suficientes para la recuperación de los recursos utilizados y que en épocas pasadas han sido satisfechas por transferencias y subvenciones del Estado español y de la Unión Europea.

En relación a los costes indicaremos que una característica muy importante relacionada con los costes de los servicios de captación, abastecimiento, recogida y saneamiento de aguas es que el porcentaje más alto de sus costes provienen de las amortizaciones de las inversiones realizadas ${ }^{38}$. De ahí la importancia de la provisión y el peligro de no tener en cuenta la recuperación de los costes, pues aunque en el momento actual se pueda hacer frente a los costes variables con unas tarifas laxas, llegará un momento en el que se va a tener que realizar una reposición o inversión que va a carecer de los recursos financieros necesarios. La ciudadanía tiene que estar dispuesta, concienciada y confiada a pagar más para asegurar la reposición futura del servicio y la regeneración medioambiental. Al mismo tiempo, no resulta fácil aproximarse a la vida útil o al valor de reposición en su caso de estas macroestructuras; instalaciones que los consumidores finales no perciben pero que, tarde o temprano, habrá que renovar, y esto provoca que existan grandes divergencias o distintos puntos de vista sobre el tratamiento de estos aspectos. Por ejemplo, desde un punto de vista económico-financiero, la tarifa debería tener un altísimo porcentaje de cuota fija similar al de los costes del servicio, cuando en realidad tiene un alto porcentaje variable en función del consumo; de ahí la asimetría de la tarifa con respecto a los costes de este servicio. En el actual periodo de crisis, todo esto se agrava, cada vez se manifiestan más las situaciones en las que los relevantes incrementos porcentuales de las tasas, debido a lo bajas que eran, siguen sin poder cubrir parte más o menos significativa de la recuperación de los costes originados por los servicios prestados.

En cuanto a los costes medioambientales y los relativos a los recursos naturales, citados en los principios básicos de la DMA, entendiendo éstos según el enfoque ecointegrador, (es decir, el coste de restauración de los deterioros ocasionados y el coste de obtención del agua), nos encontramos con que no se puede adaptar una metodología de cálculo de costes de objetos indeterminados como es el deterioro ambiental y el agua. Aunque, el agua como recurso, se pueda diferenciar de los ecosistemas vinculados a él, la calidad y cantidad de este recurso es un factor determinante en los ecosistemas asociados. Por ello, el coste ambiental del agua debería ser un coste de reposición parcial asociado al logro de objetivos de calidad acordados como de "buen estado"39. En todo caso, en el estudio realizado en Gipuzkoa, ninguna de las entidades locales integra acciones de restauración ambiental como un coste más del servicio de aguas, éstas son partidas generalmente supramunicipales destinadas al desarrollo del plan hidrológico.

\footnotetext{
36 ALTO COMISIONADO DE LAS NACIONES UNIDAS PARA LOS DERECHOS HUMANOS (2011).

37 LA ROCA Y FERRER (2007).

38 PRIETO, ZoFíO Y ÁlVAREZ (2009).

39 NAREDO (2007).
} 
En el primer Informe sobre el Desarrollo de los Recursos Hídricos en el Mundo (2003) se menciona expresamente la necesidad de mejorar la capacidad de autoevaluar y reconocer los problemas relacionados con el estado de sus recursos hídricos,

"Los profesionales del agua necesitan una mejor comprensión del amplio contexto político, económico y social, y los políticos necesitan estar mejor informados de los asuntos relacionados con los recursos hídricos" ${ }^{\prime 0}$.

Eso significa que los flujos de información, análisis, debate y consenso entre los distintos responsables de los servicios e institucionales deben prevalecer a la hora de buscar un equilibrio entre las necesidades de los ciudadanos y los aspectos medioambientales.

\section{EL CICLO INTEGRAL, EL MODELO ORGANIZATIVO Y LAS BUENAS PRÁCTICAS EN GIPUZKOA}

El ciclo integral del agua supone una sucesión de las siguientes actividades: captación; tratamiento de potabilización (ETAP); recogida o almacenamiento en depósito; distribución del agua; saneamiento; depuración (EDAR); y, vertido al río o al mar.

Estas actividades se agrupan en dos tipos de sistemas o redes: red de Alta (o primaria), y red de Baja (o secundaria).

La red de Alta agrupa tanto el abastecimiento del agua desde embalses y presas mediante una amplia red de canales y tuberías a los depósitos municipales, como el saneamiento en Alta, que incluye la infraestructura para la recogida en colectores, depuración y vertido del agua residual al medio natural.

La red en Baja supone por un lado, la distribución del agua potable a domicilios urbanos, zonas industriales y casas rurales y, por otro lado, el saneamiento, la recogida de las aguas residuales, pluviales e industriales, a través del alcantarillado del municipio. Es decir, la red en Baja consta de abastecimiento en Baja y saneamiento en Baja.

ALTA: captación,

potabilización, depósito
BAJA: distribución del agua potable y saneamiento
ALTA: recogida,

depuración y vertido

Aunque la gestión de los servicios de agua es una competencia municipal ${ }^{41}$, es importante señalar que, salvo en algún caso excepcional, en Gipuzkoa la gestión en Alta está cedida a consorcios o mancomunidades; en bastantes casos se cede incluso la gestión del ciclo integral.

Tanto los consorcios como las mancomunidades son formas asociativas para la prestación conjunta de servicios. La diferencia fundamental es a nivel organizativo o jurídico. Las mancomunidades son asociaciones de municipios para la prestación de servicios, que siendo competencia municipal se ofrecen de manera común, mediante la creación de una entidad local, con presupuesto propio y órganos de gestión diferenciados. Mientras que en los consorcios intervienen administraciones de distinto ámbito normativo; por ejemplo, diputaciones además de ayuntamientos ${ }^{42}$.

Nuestro estudio se concreta en el Territorio Histórico de Gipuzkoa, que forma parte de la Comunidad Autónoma Vasca y donde la gestión del agua está supeditada a la normativa y directrices emanadas por las

\footnotetext{
40 UNESCO (2006).

41 Artículos 25 y 26 de la LRBRL.

42 LOZANO Y LOZANO (2008)
} 
instituciones autonómicas, y en especial a la Agencia Vasca del Agua (URA). En consecuencia, debemos mencionar las características de los siguientes entes que son las que tienen la competencia junto a los ayuntamientos de la prestación de este servicio: la Mancomunidad de Aguas de Añarbe, la Mancomunidad de Aguas de Txingudi y el Consorcio de Aguas de Gipuzkoa ${ }^{43}$.

Así, la Mancomunidad de Aguas del Añarbe y su entidad pública de gestión, Aguas del Añarbe-Añarbeko Urak, S.A (AGASA), tiene como objeto la prestación de los servicios de abastecimiento y saneamiento de agua a la comarca de Donostialdea, con una población de más de 300.000 habitantes.

La Mancomunidad de Servicios de Txingudi tiene su ámbito de actuación en la comarca de Bidasoa, constituida por los municipios de Irún y Hondarribia con una población de 78.000 habitantes. Entre sus principales funciones está la gestión del ciclo integral del agua, abastecimiento y saneamiento. Incluye también la recogida de los residuos urbanos y su posterior transporte para su tratamiento, y la limpieza viaria, además de obtener energía renovable derivada de su propia labor, como actividad complementaria.

El Consorcio de Aguas de Gipuzkoa, en el que participa la Diputación Foral de Gipuzkoa además de 72 municipios, presta el servicio de abastecimiento y saneamiento de agua a alrededor de 320.000 ciudadanos. Como se puede observar la distribución de los municipios en las mancomunidades y el consorcio es territorial o geográfico.

Mientras la Mancomunidad de Aguas de Añarbe únicamente ofrece la gestión de Alta, -lo que implica que las inversiones y mantenimiento de las canalizaciones en Baja son competencia de cada ayuntamiento-, el Consorcio de Aguas de Gipuzkoa y la Mancomunidad de Aguas de Txingudi ofrecen el servicio integral de agua. En realidad el Consorcio de Aguas de Gipuzkoa presta sus servicios bien en Alta o bien integral en función de las solicitudes realizadas por los ayuntamientos de su marco de influencia.

En este sentido, el servicio de agua de los 88 ayuntamientos de Gipuzkoa se puede organizar de la siguiente manera, teniendo en cuenta la cesión o no de competencias de los municipios al consorcio o a las mancomunidades:

1. Municipal.

2. Cesión parcial de abastecimiento y/o saneamiento (red de Alta) a la Mancomunidad de Añarbe.

3. Cesión al Consorcio:

3.1. Parcial de abastecimiento y/o saneamiento (red de Alta).

3.2. Gestión de ciclo integral44.

4. Gestión de ciclo integral a la Mancomunidad de Txingudi

Es interesante observar la evolución de la clasificación organizativa de los municipios guipuzcoanos en gestión de Alta y en gestión integral, debido a la tendencia clara a favor de la segunda. Los datos que muestran este progreso se resumen en la siguiente tabla:

43 DePARTAMENTO de ORdenACIÓN DEL TERRITORIO Y MEDIO AMBIENTE DEL GOBIERNO VASCO (2005)

44 En algunos casos, el consorcio además de realizar servicios de abastecimiento y/o saneamiento de red Alta, ha realizado la gestión de abonados que consiste principalmente en la lectura de dispositivos de aforo, confección y cobro de facturas, contratación y suministro y atención a clientes, incluso el mantenimiento de algunas de las redes de saneamiento en Baja, sin llegar a ser gestión integral. 
TABLA 1: EVOLUCIÓN DE MUNICIPIOS ADHERIDOS A LA TIPOLOGÍA ORGANIZATIVA DE GESTIÓN DEL AGUA EN GIPUZKOA PERIODO 2005-2013

\begin{tabular}{|c|c|c|c|c|c|c|c|c|c|c|c|c|c|}
\hline & & \multicolumn{4}{|c|}{2005} & \multicolumn{4}{|c|}{2007} & \multicolumn{4}{|c|}{2013} \\
\hline \multicolumn{2}{|c|}{ Modelo organizativo } & \multicolumn{2}{|c|}{$\begin{array}{c}\mathbf{N}^{\circ} \\
\text { municipios }\end{array}$} & \multicolumn{2}{|c|}{ Población } & \multicolumn{2}{|c|}{$\begin{array}{c}\mathbf{N}^{\circ} \\
\text { municipios }\end{array}$} & \multicolumn{2}{|c|}{ Población } & \multicolumn{2}{|c|}{$\mathrm{N}^{\circ}$ municipios } & \multicolumn{2}{|c|}{ Población } \\
\hline \multirow{2}{*}{\multicolumn{2}{|c|}{$\begin{array}{l}\text { 1.Municipal } \\
\text { 2.Añarbe (Alta) }\end{array}$}} & 8 & $9,1 \%$ & 3.144 & $0,5 \%$ & 7 & $8,0 \%$ & 2.914 & $0,4 \%$ & 4 & $4,5 \%$ & 1.234 & $0,2 \%$ \\
\hline & & 10 & $11,4 \%$ & 304.692 & $44,2 \%$ & 10 & $11,4 \%$ & 306.102 & $44,0 \%$ & 10 & $11,4 \%$ & 312.583 & $43,8 \%$ \\
\hline \multirow{3}{*}{ 3.Consorcio } & Alta & 38 & $43,2 \%$ & 139.575 & $20,3 \%$ & 34 & $38,6 \%$ & 120.553 & $17,3 \%$ & 25 & $28,4 \%$ & 83.919 & $11,8 \%$ \\
\hline & Integral & 11 & $12,5 \%$ & 67.442 & $9,8 \%$ & 26 & $29,5 \%$ & 126.514 & $18,2 \%$ & 45 & $51,1 \%$ & 205.788 & $28,8 \%$ \\
\hline & $\begin{array}{l}\text { Alta }+ \\
\text { abonados }\end{array}$ & 19 & $21,6 \%$ & 98.407 & $14,3 \%$ & 9 & $10,2 \%$ & 62.219 & $9,0 \%$ & 2 & $2,3 \%$ & 32.386 & $4,5 \%$ \\
\hline \multicolumn{2}{|c|}{ 4.Txingudi (integral) } & 2 & $2,3 \%$ & 75.448 & $11,0 \%$ & 2 & $2,3 \%$ & 76.642 & $11,0 \%$ & 2 & $2,3 \%$ & 77.908 & $10,9 \%$ \\
\hline \multicolumn{2}{|l|}{ TOTAL } & 88 & & 688.708 & & 88 & & 694.944 & & 88 & & 713.818 & \\
\hline
\end{tabular}

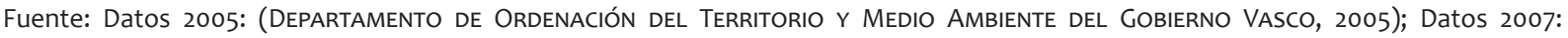

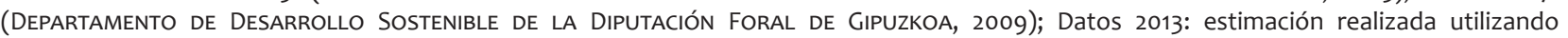
"Recuperación de Costes de los Servicios de Agua en la CAPV" y https://www.gipuzkoakour.com/Oficina-Virtual/municipios.aspx

Se puede apreciar que el Consorcio de Aguas de Gipuzkoa proporciona el servicio de Agua a 72 municipios de los 88 , abarcando más del $80 \%$ del Territorio Histórico y el $45 \%$ de la población residente, mientras que Añarbe presta el servicio en Alta a 10 municipios, incluida la capital de Donostia-San Sebastián, y por tanto abastece de agua a casi el 44\% de la población de Gipuzkoa. El resto corresponde a la Mancomunidad de Txingudi.

GRÁFICO 1: DISTRIBUCIÓN DEL PORCENTAJE DE POBLACIÓN ATENDIDA POR CADA TIPOLOGÍA ORGANIZATIVA DE GESTIÓN DEL AGUA EN GIPUZKOA, AÑO 2013

\section{Población 2013}

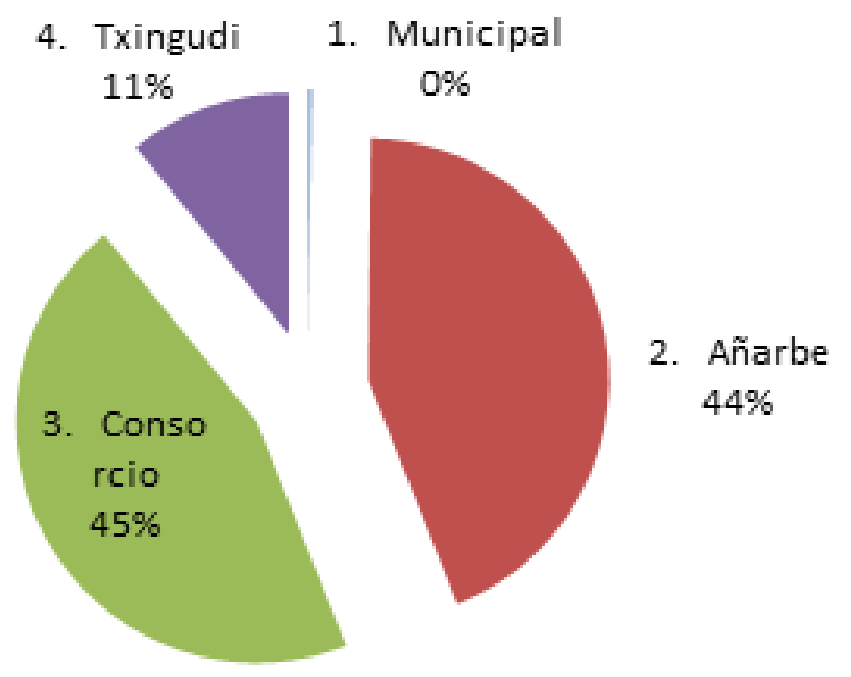

Tal y como hemos comentado, existen diferencias en cuanto al tipo de servicio que ofrece cada ente. Las mancomunidades no han modificado su modelo de servicio, pero en cambio se observa una evolución creciente en el número de municipios que contratan la prestación del servicio integral al consorcio de aguas de Gipuzkoa. 


\section{№ de municipios en cada tipología de servicio}

\section{Municipal}

3. 1 Consorcio (alta)
2. Añarbe (alta)

3.2 Consorcio (integral)

\author{
3.3 Consorcio (alta +abonados) 1 4. Txingudi (integral)
}

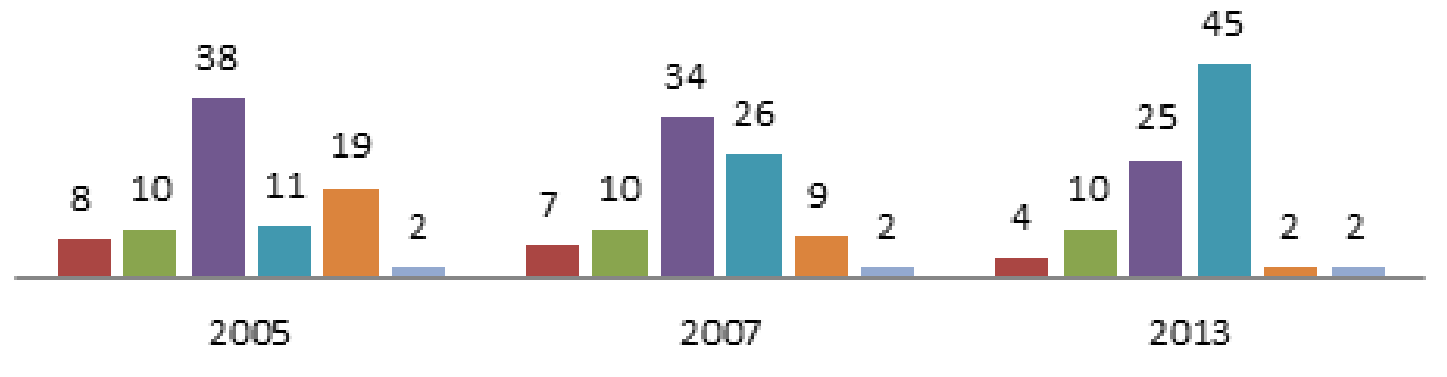

Este progreso no es fruto de la casualidad y es interesante detallar, además de las correctas actuaciones, las diferentes buenas prácticas llevadas a cabo por las diferentes entidades gestoras, haciendo hincapié en los apartados comentados anteriormente:

1. Transparencia y rendición de cuentas: cabe destacar el primer puesto obtenido por URA-Agencia Vasca del Agua según el Índice de Transparencia en la Gestión del Agua (INTRAG) 2013 elaborado por la organización Transparencia Internacional España, extensible a las entidades gestoras y la actitud de transparencia ofrecida en las entrevistas realizadas. Aunque tal y como indican todavía quedan aspectos que mejorar, la respuesta está siendo muy positiva por parte de la ciudadanía que percibe un acercamiento institucional y es cada vez más consciente de la problemática asociada a este servicio. Señalan de una forma muy positiva las posibilidades que ofrece internet para poder ofrecer y poner a disposición de la ciudadanía toda una batería de recursos informativos, documentos oficiales, de formación, de tramitación, de control, el plan hidrológico, etcétera ${ }^{45}$.

2. Fomentar la participación y concienciación de la sociedad guipuzcoana sobre la importancia del agua como recurso vital y como bien social y medioambiental: es el primero de los ocho objetivos mencionados en las Directrices sobre el uso sostenible del agua en Gipuzkoa ${ }^{46}$, en él se proponen cuarenta y una actuaciones que desarrollan este objetivo directa o indirectamente. se apuesta por las iniciativas de fomentar la divulgación, formación y sensibilización ambiental, para favorecer la adquisición de conocimientos y fomentar un cambio de mentalidad, como baluarte de la sostenibilidad ambiental. Se trata de integrar todos los elementos y factores, que intervienen en el ciclo hidrológico y fomentar la educación ambiental como estrategia prioritaria para involucrar a la sociedad en un planteamiento de conservación y recuperación del medio hídrico, acorde con los planteamientos de la DMA y los principios de la Nueva Cultura del Agua47.

3. Gestionar de manera sostenible la demanda de agua, favoreciendo políticas de control y ahorro del uso del agua con la finalidad de que se necesite la captación de menos recursos hídricos. Se toman medidas para que la población realice un uso sostenible del agua. Suele resultar difícil el cambio de hábitos y, a veces, medidas indirectas que afectan económicamente al ciudadano, como es la fijación de un consumo

45 http://www.uragentzia.euskadi.eus/

46 IKAUR y EKOLUR (2007)

47 http://www.uragentzia.euskadi.eus/ 
medio por habitante empadronado que sirva de referencia al usuario, pueden ser útiles, gravándose en ese caso en mayor importe los consumos que superen esa cifra. En Gipuzkoa, Oñati es uno de los municipios que se ha sumado a este sistema de tarificación.

4. Control: se va avanzando en la divulgación de cada vez más indicadores de gestión, pero los propios gestores consideran que hay que actuar con prudencia, y que debido a las desiguales características y múltiples formas de gestión, la interpretación de los datos de estos indicadores y el uso mediático que se pueda hacer de esa información puede ser más perjudicial que beneficioso, recordando que toda esta batería de indicadores están en manos del consejo de administración formado por delegados de las entidades locales a los que se da el servicio. Del mismo modo, es destacable la implementación, por parte de la Mancomunidad de Txingudi, del proyecto Erlea, un modelo de gestión integral de sus áreas de actividad, donde las herramientas más destacables son un Sistema de Información Geográfica y una red fija y estable de transmisión de datos, estas posicionan geográficamente toda la información de la empresa y permite obtener lecturas automáticas, de consumos y de caudales de recogida de aguas residuales, dirigidas al control diario de consumos, fugas y filtraciones para evitar despilfarros. Aunque esta inversión no influye a corto plazo en el resultado económico ni positiva ni negativamente, supone un gran avance en el control del ciclo integral del agua.

\section{LOS COSTES DEL AGUA EN GIPUZKOA Y SU RECUPERACIÓN ECONÓMICA}

\subsection{Los costes}

Una vez analizado el entorno en el que se desenvuelve el servicio del agua en la Administración Local de Gipuzkoa, vamos a centrarnos en el estudio de costes que presentan las tres instituciones (el consorcio y las mancomunidades), para posteriormente introducirnos en el establecimiento de las tasas como forma de recuperación de los costes.

Las tres entidades basan sus estudios de costes de la misma forma que las empresas, es decir, no acuden a la información presupuestaria para realizar sus cálculos, sino que implantan un sistema de costes de la misma forma que lo haría una empresa privada. Las herramientas utilizadas por los órganos decisorios de las empresas son utilizadas aquí por estas organizaciones que proporcionan servicios públicos. Por tanto, en los tres casos la entrada de datos al sistema se realiza a través del análisis de la Cuenta de Pérdidas y Ganancias y no a través de la liquidación presupuestaria como suele ser habitual en cualquier administración pública local a la hora de calcular los costes de los servicios que presta a sus ciudadanos ${ }^{48}$.

Para una mejor comprensión de la metodología utilizada y de los aspectos relevantes que condicionan el coste del servicio del agua se va a realizar un análisis conjunto de todas las entidades, indicando aquellas particularidades que por su singularidad merecen destacarse.

Los servicios que se prestan a los ciudadanos difieren según sea la institución que la proporcione, pudiéndose ofrecer un servicio Integral, como la Mancomunidad de Txingudi y el Consorcio, como parcial, normalmente en Alta, como la Mancomunidad del Añarbe y el Consorcio, el cual puede proporcionar un servicio "a la carta" según lo solicite el ayuntamiento correspondiente.

Para poder realizar un estudio comparativo adecuado en las entidades que no proporcionan el servicio integral como es la Mancomunidad de Añarbe y el Consorcio en algunas localidades, se deberían estudiar los costes en Baja de éstas para poder realizar un estudio completo de todo el Ciclo del Agua.

Debemos indicar que los estudios de costes no son homogéneos pues cada institución realiza sus estimaciones en función de la metodología que considera más adecuada, realizando interpretaciones que no suelen ser extensibles, por su subjetividad y falta de armonización, al resto de instituciones. Por tanto, la homogenización de la metodología, de los conceptos utilizados y de los criterios de valoración elegidos en todos los servicios públicos, y más en concreto en el que es objeto de este análisis, debería ser prioritaria de cara al futuro.

No obstante, se puede afirmar que al ser la mayor parte un servicio que se presta en exclusiva (excepto en la Mancomunidad de Txingudi) y proporcionados por un organismo supralocal con forma jurídica diferenciada de las corporaciones locales, la mayor parte de sus costes son directos. La utilización de "full-costing" o costes completos no conlleva mayor complejidad siendo el modelo de costes implantado en las tres instituciones, cargando al portador de costes todos los costes originados en la entidad.

48 CARRASCO ET AL. (2011) 
En relación a Txingudi, aunque se presta también el servicio de Recogida de Basuras, la mayor parte de los costes están bien identificados con los servicios (más del 80\%), y sólo algunos costes indirectos, fundamentalmente de dirección y gestión, de poca importancia relativa en el conjunto de los costes, puede conllevar alguna dificultad en su reparto. Con ello Txingudi se evita una de las dificultades más importantes que suele surgir en el ámbito empresarial como es el de la elección de la clave de reparto más adecuada para distribuir los costes indirectos, y que mejor represente la relación causa-efecto entre el coste surgido y la unidad de obra que lo puede representar. Además desde la óptica del cálculo de coste, sistemas más recientes como el ABC (Costes Basado en las Actividades) que empiezan a ser utilizadas en algunas entidades locales ${ }^{49}$, no tienen razón de ser en estos análisis.

Tal y como se ha indicado anteriormente, la mayor parte de los costes suelen ser fijos, pues conceptos como amortizaciones y personal, por ejemplo, son costes que permanecen a lo largo del tiempo y no dependen de la actividad generada. Este es un gran contratiempo cuando no se deba o pueda, trasladar al usuario final en su totalidad, o en gran parte, ese coste.

Las inversiones en infraestructuras que se realizan para esta clase de servicio suponen un gran desembolso económico y al mismo tiempo repercuten directamente en el cálculo de las amortizaciones, y posteriormente, en su reflejo en las tasas.

La primera dificultad que se plantea es el valor base que va a servir de referencia para la estimación de la amortización. La utilización como base de cálculo de los Costes Históricos o Costes de Reposición adquiere aquí una especial relevancia, y suele ser un tema controvertido pues se plantea cuál es la finalidad de las tasas, si recuperar la inversión que se realizó o poder hacer frente con esos ingresos a las necesidades de renovación de las infraestructuras. Las amortizaciones suelen suponer en todos los casos más de un 20\% de los costes de los servicios (y en algunos casos y dependiendo de las inversiones realizadas y de su forma de cálculo, más de un 30\%), de ahí la importancia de una valoración adecuada.

El coste de mantenimiento también es un aspecto relevante en esta clase de servicio planteándose en cada caso la potenciación del aspecto preventivo o el correctivo, buscándose un equilibrio entre esos posicionamientos que repercuten en los costes correspondientes. Ya no es tanto la dificultad en la imputación de los costes como en la gestión de los inmovilizados de la entidad para su mejor eficiencia.

Entre el coste de personal, amortizaciones y mantenimiento se llevan en todos los casos más de $70 \%$ de los costes totales del Ciclo del agua.

Para hallar el coste total del suministro, canalización, depuración y saneamiento del agua en aquellos ayuntamientos con competencias en Baja no transferidas a la instituciones anteriores, se deben incorporar a los costes anteriores (que en realidad para ellos será la tasa fijada por la Mancomunidad de Añarbe o el Consorcio) sus propios costes en Baja, cuyo cálculo suele realizarse, fundamentalmente, a través de las liquidaciones presupuestarias, utilizando cada uno de ellos su propia metodología que puede ser o no coincidente. De esa forma se obtendría un coste integral que podría compararse (aunque no en su metodología) con el resto de instituciones.

Otra cuestión a considerar es la repercusión de las fugas de agua (mermas) en Alta por ineficiencia de la red de distribución, subcontaje de los instrumentos de medida, etc. Dependiendo de donde se originan tendrán unos costes u otros, complicándose la situación a la hora de plantearse su repercusión o no de ese coste a la tasa a cobrar a los usuarios.

\subsection{Las Tasas}

Si exceptuamos las ayudas recibidas por distintas instituciones públicas (Europa, Administración Central del Estado, etc.) que normalmente corresponden a transferencias de capital para la realización de grandes inversiones en la canalización o saneamiento de las aguas, la mayor parte de los ingresos relacionados con el agua vienen a través de las tasas o tarifas que se fijan a los usuarios del agua: familias, industrias, sector agrícola, etc. En algunos casos, como Txingudi, aparte de los ingresos normales relacionados con el suministro y depuración del agua también existen ingresos internos procedentes de la creación de energía eléctrica que es utilizada para autoconsumo de su propio proceso integral del agua.

Aunque las fórmulas son distintas a la hora de establecer las tarifas en las 3 instituciones, sin embargo, las variables que las condicionan suelen ser las mismas, desdoblándose en una parte fija y otra variable. Las referencias

49 PRIETO Y ROBLEDA (2006) 
para poder fijarlas suelen ser la DMA, las recomendaciones de AEAS (Asociación Española de Abastecimiento de Agua y Saneamiento), así como la Ley de Aguas que establece que la,

“Administración con competencias en materia de suministro de agua establecerá las estructuras tarifarias por tramos de consumo, con la finalidad de atender las necesidades básicas a un precio asequible y desincentivar los consumos excesivos".

Las tarifas tienen en la parte fija dos conceptos que cubrir: el abastecimiento y, la recogida de aguas residuales, su transporte y depuración.

La parte variable se calcula en función del consumo realizado y del tipo de usuario (Doméstico y No Doméstico), desglosándose en 3 conceptos o servicios: abastecimiento, saneamiento y depuración. En algunas instituciones se tienen en cuenta el diámetro de tubería contratado u otras unidades de medida, representativas del comportamiento de los costes, y que sirven, por tanto, para fijar las tarifas en su parte variable como son el número de habitantes y la superficie urbanizada.

Mientras Txingudi realiza el ciclo integral y establece el precio al usuario finalista, en Añarbe, y en algunos casos del Consorcio (en los ayuntamientos que sólo reciben servicios de Alta) se fija una tasa (que podría considerarse como "precio de transferencia o cesión") al Ayuntamiento para que éste incorpore posteriormente los costes en Baja, dando como resultado la tarifa final que se cobra al usuario finalista. Esta diferencia es fundamental tanto para la gestión del servicio como para la mayor libertad en la toma de decisiones, tanto de inversiones a realizar como de la fijación de la tasa final. Normalmente en aquellos ayuntamientos que proporcionan servicio en Baja hay una mayor influencia en la negociación del importe que van a cobrar las instituciones. Por tanto, la controversia está en si esos costes son aceptados por el órgano responsable a la hora de fijar la tarifa o precio público, pues en este caso son los responsables de las administraciones locales los que debe considerar la repercusión del precio en la ciudadanía.

El gran problema de los costes es poder repercutir en las tarifas el coste de las amortizaciones para hacer frente a las nuevas inversiones, aunque previamente se incorporen, y así se hace, en el coste del servicio. Los razonamientos para la no inclusión en las tarifas suelen ser muy diversos: desde que algunas de las infraestructuras han sido subvencionadas por otras instituciones (Europa, Administración Central, Gobierno Autonómico, etc.) y por tanto, no es necesario repercutirlas a los usuarios, hasta que las infraestructuras ya están amortizadas. En el primer caso, habría que decir que no todas las infraestructuras han sido financiadas por otras instituciones y, aunque así sea, debe tenerse en cuenta que tarde o temprano esas infraestructuras deberán de ser modificadas y alguien deberá haber previsto esa situación para no poner en peligro financiero la propia prestación del servicio, y más si tenemos en cuenta que las distintas administraciones públicas cada vez son más reacias a financiar con sus propios presupuestos esas inversiones. Este último argumento es extensible a la segunda cuestión planteada.

En la Mancomunidad de Txingudi incluso temporalmente durante unos años (hasta el 2008) se incorporó en la tarifa un importe extraordinario para cubrir la financiación de las nuevas infraestructuras de saneamiento hasta cubrir el importe de la inversión, desapareciendo a partir de entonces esa derrama excepcional, aunque el coste de esas inversiones u otras se incorporan como un elemento más dentro del servicio específico que las utiliza.

Estudiando las tarifas que se fijan en las tres instituciones, sin duda, en la que más dispersión existe es en la Mancomunidad de Añarbe. Fundamentalmente se debe a que su estimación se basa en el cálculo de dos costes con metodologías, fuentes de información e instituciones diferenciadas, aparte de los intereses particulares de la corporación local. Al contrario, en las que prestan un servicio integral, esas divergencias no suelen existir.

Por último, indicaremos que un posicionamiento u otro pueden conllevar repercusiones en los presupuestos locales. El interés fundamental del precio que se carga a los ciudadanos es conocer el grado de cobertura, o de financiación, que tiene el servicio con los ingresos que se perciben por el usuario de ese servicio. El resto de los costes no asumidos por el "cliente" deberán ser cubiertos vía presupuestos a través de otros ingresos, normalmente tributarios, no vinculados directamente con ese servicio.

\section{CONCLUSIONES}

El agua es un servicio público imprescindible que proporcionan las administraciones locales a sus ciudadanos. Este servicio se presta diferenciando varias fases: Abastecimiento y Saneamiento tanto en Alta como en Baja, siendo cada entidad local la que decide si proporciona con sus propios medios ese ciclo integral o delega en 
entidades supralocales parte o la totalidad del servicio. Esa delegación se realiza en aras a una mejor gestión de los recursos hidráulicos tanto en la fase de abastecimiento de agua potable como en el saneamiento del agua utilizada. En el caso del Territorio Histórico de Gipuzkoa existen tres entidades que realizan esa función total o parcialmente, como son las Mancomunidades de Añarbe y de Txingudi y el Consorcio de Gipuzkoa.

Lo anteriormente expuesto provoca que los estudios que se puedan realizar para poder comparar los costes entre las distintas poblaciones y las tarifas sean prácticamente imposibles. Fundamentalmente hay dos motivos:

- El primero es debido a la utilización, en bastantes casos, de cálculos de costes en función de la Cuenta de Pérdidas y Ganancias (las 3 entidades supralocales citadas) y en otras situaciones se mezcla esa Cuenta con las liquidaciones presupuestaria, si el ayuntamiento presta alguno de los servicios relacionados con el agua. Las fuentes informativas son distintas y los conceptos y criterios de valoración consiguientemente suelen también serlos.

- El segundo se debe a la incorporación o no en cada caso de las amortizaciones de las infraestructuras y el valor de referencia escogido, histórico o de reposición (dependiendo de la antigüedad de las infraestructuras puede llegar a ser más de un 50\% entre la elección de un criterio de valoración u otro), en el caso de que se incorpore, también provoca que los costes no puedan ser comparables entre las distintas localidades. La argumentación que en muchos casos se esgrime desde la óptica del analista de presupuestos es que este concepto no supone salida de dinero, no está recogido en los presupuestos, y por tanto no debe recuperarse vía ingresos.

Igualmente hay distintas formas de calcular las tasas de agua, dependiendo en gran parte de quién proporciona el servicio, y por otro lado de la injerencia política a la hora de poner un mayor o menor precio dependiendo en bastantes casos de otras circunstancias ajenas a la recuperación de los costes. Al ser un servicio público suele haber una visión distinta desde el punto de vista del gestor o responsable del servicio proporcionado, del político, como responsable público, cabeza visible ante la ciudadanía y que debe rendir cuentas periódicamente de sus actos, que es quien tiene la competencia de fijar en último término el precio que se cobrará a sus conciudadanos. Teniendo en cuenta el proceso de la toma de decisión en el establecimiento de la tasa de los servicios de las aguas, el cálculo del coste de estos servicios puede considerarse como suficiente en tanto en cuanto las tasas no superan sus costes. Ahora bien, es totalmente insuficiente a la hora de valorar aspectos relacionados con la recuperación de costes, reposición de infraestructuras, regeneración, eficiencias de pérdidas y filtraciones. En general, consiguientemente, las tasas relacionadas con los servicios de agua no son suficientes, en sí mismas, para hacer frente a las futuras inversiones y tal y como están establecidas no garantizan su sostenibilidad.

El análisis anterior provoca que sea inviable poder realizar un estudio comparativo que pueda conllevar a un análisis posterior de la eficacia y eficiencia de los recursos utilizados en ese servicio. Es obvio, además, que para realizar un estudio comparativo en condiciones se deberían considerar otros aspectos como son los geológicos, tipología de las urbanizaciones y viviendas, distribución geográfica y demográfica o la composición de los sectores económicos relacionados con cada municipio. De todo ello se deduce la prudencia y escasa divulgación de indicadores relativos a la gestión del ciclo integral del agua.

Las entidades gestoras del agua gozan de buen prestigio, y el grado de transparencia es cada vez mayor habida cuenta de la información que se proporciona a la ciudadanía. Sin embargo, los informes publicados van más dirigidos a que los usuarios aumenten su sensibilización ambiental y realicen un consumo sostenible, que a reflejar indicadores de gestión de las propias organizaciones.

A pesar de ello, no debe ser excusa para fomentar y potenciar la proactividad en el uso eficiente del agua con instrumentos de control de los malos usos, abusos, filtraciones o fugas como las de la mancomunidad de Txigudi; con políticas de concienciación de un consumo responsable como las del municipio de Oñati, que vayan de la mano de altos índices de participación ciudadana y consenso democrático y, sobre todo potenciar la confianza en las actuaciones de los gestores a través de altos índices de transparencia hacia la ciudadanía, que conllevará una mejor comprensión de las decisiones adoptadas por los responsables institucionales y una mayor involucración de la población en la correcta gestión de este servicio.

Por último, según se vaya avanzando en la sensibilización y la concienciación, habrá que incidir en optimizar la gestión de los recursos públicos mediante indicadores más o menos estandarizados o normalizados que faciliten una toma de decisiones racional, el seguimiento de los objetivos conseguidos y el análisis de las desviaciones. Con ello, sin duda incrementará la confianza de la ciudadanía dispuesta a hacer frente a la recuperación de todos los costes citados en la DMA. 


\section{BIBLIOGRAFIA}

AGUAS del AÑARBE - AÑARBEKo URAK S.A.: http://www.agasa.es/

Alto Comisionado de las Naciones UnidAs PARA los Derechos Humanos (2011). "El derecho al agua", Folleto informativo $n^{\circ} 35$.

ARrojo AGUdo, PedRo (2004). (Coordinador) El agua en España: propuestas de futuro (1 $1^{\text {a }}$ edición) Ediciones del Oriente y del Mediterráneo.

\section{ASOCIACIÓn ESPAÑOLA DE ABASTECIMIENTOS DE AGUA Y SANEAMIENTO (AEAS): http://www.aeas.es/}

BALL, AMANDA Y GRUBNIC, SUZANA (2007). "Sustainability accounting and accountability in the public sector", en: UNERMAN, JeFFREY; BEBbINGTON, JAN Y O'DWYER, BRENDAN (EDS), Sustainability Accounting and Accountability. London, Routledge, pp. 243-265

Bebbington, Jan; Larrinaga González, Carlos. y Moneva Abadía, Jose M. (2008) "Corporate Social Responsibility Reporting and Reputation Risk Management", Accounting, Auditing and Accountability Journal, Vol. 21, n³, pp. 337-361.

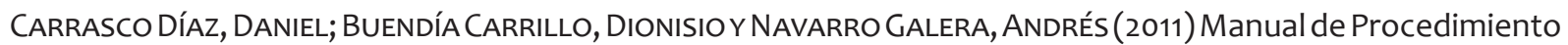
para la Implantación de un Sistema de Costes en la Administración Local (MAPISCAL)

CHAN, JAMES L. (2003). "Government accounting: an assessment of theory, purposes and standards", Public Money \& Management, Vol. 23, $\mathrm{n}^{\circ}$ 1, pp. 13-20.

COMISIÓN EUROPEA (2009). “Indicadores de confianza de la Comisión Europea”, Índice: revista de estadística y sociedad, $\mathrm{n}^{\circ}$ 32, pp. 21-23.

Del Villar, Alberto (2010). “Los precios de los servicios del agua. Un análisis prospectivo de demanda sobre los usos domésticos”, Estudios de economía aplicada, Vol. 28, n², pp. 333-356.

DEPARTAMENTO DE DESARROLlo SOSTENIBLE DE LA DiPUTACIÓN FORAL DE GIPUZKOA (2009). Inversiones en materia de Obras Hidráulicas realizadas por la Administración en el Territorio Histórico de Gipuzkoa. Fase Il: Análisis de las Tarifas.

Departamento de Ordenación del TerRitorio y MEdio Ambiente del Gobierno VASCO (2005). Recuperación de Costes de los Servicios de Agua en la CAPV.

DOMÈNECH, LAIA (2011). "Rethinking water management: from centralised to decentralised water supply and sanitation models”, Documents d'anàlisi geogràfica, Vol. 57, n² 2, pp. 293-310.

GIPUZKOAKO UR KONTSORTZIOA: http://www.gipuzkoakour.com/default.aspx

GRAY, ROB (2006). "Social, environmental and sustainability reporting and organizational value creation: whose value? Whose creation?" Accounting, Auditing and Accountability Journal, Vol. 19, nº 6, pp. $793-819$.

GUICHOT, EMILIO. (2014). Transparencia, Acceso a la Información Pública y Buen Gobierno. Madrid, Tecnos, pp. 383.

IKAUR y EKOLUR (2007). "Directrices sobre el agua sostenible en Gipuzkoa. Objetivos, actuaciones e indicadores" Diputación Foral de Gipuzkoa, Departamento para el Desarrollo Sostenible.

JUÁREZ Sánchez-Rubio, CIPRIANO (2008). “Indicadores hídricos de sostenibilidad y desarrollo turístico y residencial en la Costa Blanca (Alicante)”, Boletín de la Asociación de Geógrafos Españoles, nº 47, pp. $213-243$.

Kim, PAn SuK; Halligan, John; Cho, Namshin; OH, CheOl H. y Eikenberry, AnGela M. (2005). "Toward participatory and transparent governance: report on the Sixth Global Forum on Reinventing Government”, Public Administration Review, Vol. 65, nº 6, pp. 646-654.

KLUVERS, RON (2010) “Mechanisms of Accountability in Local Government: An exploratory Study", International Journal of Business and Management, Vol. 5, $\mathrm{n}^{\circ}$ 7, pp.46-53.

La Roca Cervigón, Francesc y FerRer matVieychuc, Graciela (2007). "La economía en el desarrollo de la directiva Marco del Agua”, Observatorio medioambiental, nº 10, pp. 179-198.

LARRINAGA GONZÁLEZ, CARLOS Y Pérez CHAMORRO, VICENTE(2008). "Sustainability Accounting and Accountability 
in Public Water Companies”, Public Money \& Management, Vol. 28, n 6, pp. 337-343.

LEE, JANET (2008). "Preparing Performance Information in The Public Sector: An Australian Perspective”, Financial Accountability and Management, Vol. 24, n² 2, pp. 117-149.

lozano Valencia, Miguel angel y lozano Valencia, Peio (2008). “Las Mancomunidades de servicios, un ejemplo de vertebración territorial para Guipúzcoa: caracterización de los residuos domésticos en dicho territorio a partir de la gestión mancomunada", Boletín de la Asociación de Geógrafos Españoles, n 48, pp. $155-174$.

MORAL ITUARTE, LEANDRO DEL (2009). “Nuevas tendencias en gestión del agua, ordenación del territorio e integración de políticas sectoriales”, Scripta Nova: Revista electrónica de geografía y ciencias sociales, n 13.

Moral ItUARTe, LeANDRo del y ARrojo Agudo, PedRo (2003). (Coordinadores) La directiva marco del agua: realidades y futuros ( $1^{\mathrm{a}}$ edición) Fundación Nueva Cultura del Agua.

MuÑIz Pérez, Manuel Antonio y García Valiñas, María Ángeles (2004). “Una propuesta para la regulación de precios en el sector del agua: el caso español”, Papeles de trabajo del Instituto de Estudios Fiscales. Serie economía, $\mathrm{n}^{\circ} 1$, pp. 1-40.

MUSSARI, RICCARDO Y MONFARDINI, PATRIZIO (2010). "Practices of social reporting in public sector and nonprofit organizations”, Public Management Review, Vol. 12, n 4, pp. 487-492.

NAREDO PÉREZ, JosÉ MANUEL (2006). “La encrucijada de la gestión del agua en España”, El agua en el siglo XXI: gestión y planificación ( $1^{\text {a }}$ ed., pp. 9-28) Institución Fernando el Católico.

NAREdo PÉREZ, José MANuel (2007). “Costes y cuentas del agua”. Propuestas desde el enfoque ecointegrador Ponencia marco del Seminari sobre costos i comptes de l'aigua a Catalunya en relació amb la Directiva marc de l'aigua, Generalitat de Catalunya.Departament de Medi Ambient i Habitatge, Barcelona, Vol. 18.

ORDÓÑEZ DE HARO, CARMINA Y BRU MARTíNEZ, LLUÍS (2003). “Análisis de la privatización y regulación del servicio de abastecimiento de agua en Málaga”, Cuadernos de Ciencias Económicas y Empresariales, n 44, pp. 81-98.

PINA, VICENTE; TORRES, LOURDES Y YETANO, ANA. (2009). "Accrual Accounting in EU local governments: one method, several approaches”, European Accounting Review, Vol. 18, n 4, pp. 765-807.

Prieto GuiJARro, Ángel; Zofío Prieto, José luis y Álvarez Ayuso, InMACUlada (2009). “Economías de escala, densidad y alcance en la provisión pública de infraestructura básica municipal”, Hacienda pública española, n 190, pp. 59-94.

Prieto Martín, Carlos y Robleda Cabezas, Helio (2006). (Coordinadores) Guía para la implantación de un sistema de costes en la Administración Local. FEMP.

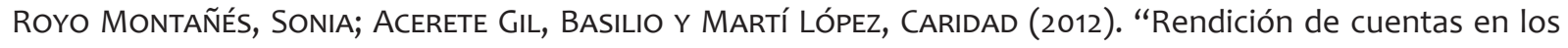
ayuntamientos españoles a través de indicadores de gestión” Presupuesto y Gasto público, n 66, pp. $183-198$.

SERVICIOS DE TXINGUDI - TXINGUDIKO ZERBITZUAK (STTZ): http://www.txinzer.com/

UNESCO. (2006). El agua una responsabilidad compartida. 2do. Informe de las Naciones Unidas sobre el Desarrollo de los Recursos Hídricos en el Mundo. Resumen Ejecutivo.

URA UR AGENTZIA - AGENCIA VASCA DEL AGUA: http://www.uragentzia.euskadi.net

WELCH, ERIC W., HiNNANT, ChARLES C. y MOON, M. JAE (2005). “Linking citizen satisfaction with e-government and trust in government", Journal of Public Administration Research and Theory, Vol. 15, n 3, pp. 371-391. 